\title{
THE INTEGRATION OPERATOR IN TWO VARIABLES
}

\author{
A. ATZMON AND H. MANOS
}

(Communicated by Palle E. T. Jorgensen)

\begin{abstract}
In this paper we consider the integration operator in two variables on $L_{2}[0,1]^{2}$, determine its multiplicity and reducing subspaces, and make some observations about its invariant and hyperinvariant subspaces.
\end{abstract}

\section{INTRODUCTION}

The purpose of this paper is to study the Volterra integration operator $W$ in two variables, that is, the operator defined on $L_{2}[0,1]^{2}$ by

$$
(W f)(x, y) \stackrel{\text { def }}{=} \int_{0}^{y} d s \int_{0}^{x} f(t, s) d t .
$$

In particular we find its multiplicity and reducing subspaces and obtain some information on its invariant and hyperinvariant subspaces. It will follow from our results that the properties of $W$ are quite different from the properties of the classical Volterra operator $V$ (defined on $L_{2}[0,1]$ by $\left.(V f)(x) \stackrel{\text { def }}{=} \int_{0}^{x} f(t) d t\right)$. It is well known that $V$ is compact and quasi-nilpotent. Since $W=V \otimes V$, the same properties are also shared by $W$. These facts are also easily verified directly.

Before describing the content of this paper, we introduce some notation and recall some definitions. For a complex Banach space $X$, we will denote by $L(X)$ the algebra of bounded linear operators on $X$. If $A$ is a subalgebra of $L(X)$ which contains the identity operator, then a subset $G$ of $X$ is called cyclic for $A$, if the linear span of the set $\{T x: x \in G, T \in A\}$ is dense in $X$. The smallest cardinality of a cyclic set for the algebra $A$ is called the multiplicity of $A$ and will be denoted by $m(A)$.

The multiplicity of an operator $T$ in $L(X)$ is defined as the multiplicity of the algebra generated in $L(X)$ by $T$ and the identity operator and will be denoted by $m(T)$.

The commutant of $T$ is defined by

$$
T^{\prime} \stackrel{\text { def }}{=}\{B \in L(X): T B=B T\} .
$$

Received by the editors February 24, 1992.

1991 Mathematics Subject Classification. Primary 47A15; Secondary 47G10.

Key words and phrases. Integration operator, multiplicity, invariant subspace, hyperinvariant subspace, reducing subspace. 
A closed subspace $M$ of $X$ is called an invariant subspace of $T$, if $T$ maps $M$ into itself. If $M$ is also invariant for every operator in $T^{\prime}$, then $M$ is called hyperinvariant for $T$. Let $\mathscr{H}$ be a Hilbert space and $T \in L(\mathscr{H})$. A closed subspace $E$ of $\mathscr{H}$ is called a reducing subspace for $T$, if $E$ and $E^{\perp}$ are both invariant under $T$.

It is well known (see [2, Theorem 4.14]) that the invariant subspaces of $V$ are exactly the subspaces $M_{a}$ of the form $M_{a}=\left\{f \in L_{2}[0,1]: f=0\right.$ a.e. on $[0, a]\}$ for some $0<a<1$. It follows from this, and it is also easily seen directly, that the function $f \equiv 1$ is cyclic for $V$; hence, $m(V)=1$. It also follows from this description of invariant subspaces that $V$ has no proper reducing subspaces. Also, since $V$ is unicellular, by a general result (see [2, Corollary 6.27]), every invariant subspace of $V$ is also hyperinvariant.

In $\S 2$ we prove that unlike $V$, the operator $W$ has infinite multiplicity.

In $\S 3$ we consider the reducing subspaces of $W$ and prove that the only such subspaces are $S_{+}$and $S_{-}$, which consist of the symmetric functions and antisymmetric functions in $L_{2}[0,1]^{2}$ respectively; that is,

$$
\begin{aligned}
& S_{+}=\left\{f \in L_{2}[0,1]^{2}: f(x, y)=f(y, x), \text { a.e. on }[0,1]^{2}\right\}, \\
& S_{-}=\left\{f \in L_{2}[0,1]^{2}: f(x, y)=-f(y, x), \text { a.e. on }[0,1]^{2}\right\} .
\end{aligned}
$$

In $\S 4$ we give some examples of invariant and hyperinvariant subspaces of $W$; however, the complete characterization of these subspaces remains open.

\section{The MUlTiplicity of $W$}

In this section we show that $W$ has infinite multiplicity, that is, we prove

Theorem 1. $m(W)=\infty$.

The proof of the theorem will be based on a result from [1, Proposition 2.1]. For the sake of completeness we include its statement and proof.

Proposition 2. Let $T$ be an operator in $L(X)$, and assume that for some integer $n \geq 2$ there exists a nonzero continuous $n$-linear mapping $\phi$ of $X^{n}$ into some topological vector space $Y$, such that, for every $n$-tuple $\left(x_{1}, \ldots, x_{n}\right)$ in $X^{n}$ for which $x_{i}=x_{j}$ for some $1 \leq i<j \leq n$ and for every pair of nonnegative integers $\left(k_{1}, k_{2}\right), \phi\left(x_{1}, x_{2}, \ldots, T^{k_{1}} x_{i}, x_{i+1}, \ldots, T^{k_{2}} x_{j}, \ldots, x_{n}\right)=0$. Then $n \leq m(T)$.

Proof. Let $A$ be the subalgebra of $L(X)$ generated by $T$ and the identity operator. First we note that since the set $D=\left\{T^{n}: n \geq 0\right\}$ spans $A$, the assumption on $\phi$ implies that for every $\left(T_{1}, T_{2}\right) \in A \times A$ and for every $n$ tuple $\left(x_{1}, \ldots, x_{n}\right)$ in $X^{n}$ for which $x_{i}=x_{j}$ for some $1 \leq i<j \leq n$

$$
\phi\left(x_{1}, x_{2}, \ldots, T_{1} x_{i}, x_{i+1}, \ldots, T_{2} x_{j}, \ldots, x_{n}\right)=0 \text {. }
$$

Let $G$ be any subset of $X$ which contains less than $n$ elements, and consider the set $M=\operatorname{span}\{S x: x \in G, S \in A\}$. The hypothesis that $G$ contains less than $n$ elements implies by (1) that $M^{n} \subseteq \operatorname{ker} \phi$, and therefore since $\phi$ is continuous, $\bar{M}^{n}={\overline{M^{n}}}_{\operatorname{lor}} \phi$. Remembering that $\phi \not \equiv 0$, we conclude that $\bar{M}^{n} \neq X^{n}$ and therefore $\bar{M} \neq X$.

In the proof of the theorem it will be convenient to write $W$ as a convolution 
operator. For $f, g \in L_{2}[0,1]^{2}$ the convolution is defined by

$$
(g \star f)(x, y) \stackrel{\text { def }}{=} \int_{0}^{y} d s \int_{0}^{x} f(t, s) g(x-t, y-s) d t .
$$

It is known and easily verified that the convolution is commutative, associative, $g \star f \in C\left([0,1]^{2}\right)$, and $\|f \star g\|_{2} \leq\|f\|_{2}\|g\|_{2}$. If we denote by $U$ the function $U \equiv 1$ on $[0,1]^{2}$ then it is clear that for every $f \in L_{2}[0,1]^{2}$, $W f=U \star f$.

In view of Proposition 2, the conclusion of Theorem 1 follows from

Proposition 3. For every $n \geq 2$ there exists an $n$-linear mapping $\phi_{n}$ that satisfies the assumptions of Proposition 2 for $W$.

Proof. For $a \geq 1$, we denote by $\square_{a}$ the rectangle $[0,1] \times[0,1 / a]$ and by $T_{a}$ the operator on $L_{2}[0,1]^{2}$ defined by

$$
\left(T_{a} f\right)(x, y) \stackrel{\text { def }}{=} \begin{cases}f(a y, x / a), & (x, y) \in \square_{a}, \\ 0, & \text { otherwise. }\end{cases}
$$

It is easily verified that $T_{a}$ is a continuous linear operator on $L_{2}[0,1]^{2}$ and that for every $(x, y) \in \square_{a}$

$$
\left(T_{a} W f\right)(x, y)=\left(W T_{a} f\right)(x, y) .
$$

Let $n>1$, and choose $n-1$ real numbers $a_{1}, a_{2}, \ldots, a_{n-1}$ such that $a_{1}=1$ and $a_{k}<a_{k+1}$ for $k=1,2, \ldots, n-2$, and define the operator $P_{n}$ on $L_{2}[0,1]^{2}$ by

$$
\left(P_{n} f\right)(x, y)= \begin{cases}f(x, y), & (x, y) \in \square_{a_{n-1}}, \\ 0, & \text { otherwise. }\end{cases}
$$

For every $f_{1}, \ldots, f_{n}$ in $L_{2}[0,1]^{2}$ consider the matrix

$$
A\left(f_{1}, f_{2}, \ldots, f_{n}\right)=\left(\begin{array}{ccc}
f_{1} & \cdots & f_{n} \\
T_{a_{1}} f_{1} & \cdots & T_{a_{1}} f_{n} \\
\vdots & & \vdots \\
T_{a_{n-1}} f_{1} & \cdots & T_{a_{n-1}} f_{n}
\end{array}\right)
$$

and define the mapping $\phi_{n}:\left(L_{2}[0,1]^{2}\right)^{n} \rightarrow L_{2}[0,1]^{2}$ by

$$
\phi_{n}\left(f_{1}, \ldots, f_{n}\right) \stackrel{\text { def }}{=} P_{n}\left\{\operatorname{det}\left[A\left(f_{1}, f_{2}, \ldots, f_{n}\right)\right]\right\},
$$

where multiplication in $\operatorname{det} A$ is convolution. Since for every $n$ functions $g_{1}, \ldots, g_{n}$ in $L_{2}[0,1]^{2}$ we have that

$$
\left\|g_{1} \star g_{2} \star \cdots \star g_{n}\right\|_{2} \leq\left\|g_{1}\right\|_{2}\left\|g_{2}\right\|_{2} \cdots\left\|g_{n}\right\|_{2},
$$

and since the operators $T_{a_{i}}$ are continuous, it follows that $\phi_{n}$ is a continuous $n$-linear mapping of $\left(L_{2}[0,1]^{2}\right)^{n}$ into $L_{2}[0,1]^{2}$. Next, let $\left(f_{1}, \ldots, f_{n}\right) \in$ $\left(L_{2}[0,1]^{2}\right)^{n}$ and assume that there exist $1 \leq i<j \leq n$ such that $f_{i}=W^{r} f$, $f_{j}=W^{m} f$ for some $f \in L_{2}[0,1]^{2}$. We have to show that $\phi_{n}\left(f_{1}, \ldots, f_{n}\right) \equiv 0$. First if $(x, y) \notin \square_{a_{n-1}}$ then, for every $f \in L_{2}[0,1]^{2},\left(P_{n} f\right)(x, y)=0$ and so

$$
\left[\phi_{n}\left(f_{1}, \ldots, f_{n}\right)\right](x, y)=\left[P_{n}(\operatorname{det} A)\right](x, y)=0 .
$$


It remains to prove that this holds also for $(x, y) \in \square_{a_{n-1}}$. By changing the order of the columns of $A$ we may assume that $i=1$ and $j=2$, namely, $f_{1}=W^{r} f$ and $f_{2}=W^{m} f$. Then on $\square_{a_{n-1}}$

$$
\begin{aligned}
& \phi_{n}\left(W^{r} f, W^{m} f, f_{3}, \ldots, f_{n}\right) \\
& =\operatorname{det}\left(\begin{array}{ccccc}
W^{r} f & W^{m} f & f_{3} & \ldots & f_{n} \\
T_{a_{1}} W^{r} f & T_{a_{1}} W^{m} f & T_{a_{1}} f_{3} & \cdots & T_{a_{1}} f_{n} \\
\vdots & \vdots & \vdots & \vdots & \\
\vdots & \vdots & \vdots & \vdots & \\
T_{a_{n-1}} W^{r} f & T_{a_{n-1}} W^{m} f & T_{a_{n-1}} f_{3} & \cdots & T_{a_{n-1}} f_{n}
\end{array}\right) .
\end{aligned}
$$

For every $1 \leq k<n-1, a_{n-1}>a_{k}$; hence, $\square_{a_{n-1}} \subset \square_{a_{k}}$. Therefore, using (3) and remembering that $W f=U \star f$ and that convolution is associative and commutative, we obtain that on $\square_{a_{n-1}}$

$$
\begin{array}{r}
\phi_{n}\left(W^{r} f, W^{m} f, f_{3}, \ldots, f_{n}\right) \\
\quad=\operatorname{det}\left(\begin{array}{ccccc}
W^{r} f & W^{m} f & f_{3} & \cdots & f_{n} \\
W^{r} T_{a_{1}} f & W^{m} T_{a_{1}} f & T_{a_{1}} f_{3} & \cdots & T_{a_{1}} f_{n} \\
\vdots & \vdots & \vdots & \vdots & \\
\vdots & \vdots & \vdots & \vdots & \\
W^{r} T_{a_{n-1} f} f & W^{m} T_{a_{n-1}} f & T_{a_{n-1}} f_{3} & \cdots & T_{a_{n-1}} f_{n}
\end{array}\right) \\
=W^{r+m} \operatorname{det}\left(\begin{array}{ccccc}
f & f & f_{3} & \cdots & f_{n} \\
T_{a_{1}} f & T_{a_{1}} f & T_{a_{1}} f_{3} & \cdots & T_{a_{1}} f_{n} \\
\vdots & \vdots & \vdots & \vdots & \\
\vdots & \vdots & \vdots & \vdots & \\
T_{a_{n-1}} f & T_{a_{n-1}} f & T_{a_{n-1}} f_{3} & \cdots & T_{a_{n-1}} f_{n}
\end{array}\right) .
\end{array}
$$

Now in the last matrix the first two columns are the same on $\square_{a_{n-1}}$ and, therefore, the determinant vanishes on $\square_{a_{n-1}}$. Noticing that if $g$ is any function that vanishes on some rectangle of the form $[0, a] \times[0, b]$-that is included in $[0,1]^{2}$-then $W^{k} g$ also vanishes on that rectangle for every $k$, we conclude that $\phi_{n}\left(W^{r} f, W^{m} f, f_{3}, \ldots, f_{n}\right)=0$ on $\square_{a_{n-1}}$.

It remains to show that $\phi_{n}$ is not identically zero. For this consider the functions $g_{1}(x, y)=1, g_{2}(x, y)=x, \ldots, g_{n}(x, y)=x^{n-1}$. We claim that $\phi_{n}\left(g_{1}, \ldots, g_{n}\right) \not \equiv 0$. Indeed, by definition (2) and the fact that $\square_{a_{n-1}} \subseteq \square_{a_{k}}$ we have for $(x, y)$ in the rectangle $\square_{a_{n-1}}$ and for every $1 \leq k \leq n-1$ that $\left(T_{a_{k}} g_{m}\right)(x, y)=g_{m}\left(a_{k} y, x / a_{k}\right)=a_{k}^{m-1} y^{m-1}$. By the definition of $\phi_{n}$ we get that on $\square_{a_{n-1}}$

$$
\phi_{n}\left(g_{1}, \ldots, g_{n}\right)=\operatorname{det}\left(\begin{array}{cccc}
1 & x & \cdots & x^{n-1} \\
1 & y & \cdots & y^{n-1} \\
1 & a_{2} y & \cdots & a_{2}^{n-1} y^{n-1} \\
\vdots & \vdots & & \vdots \\
1 & a_{n-1} y & \cdots & a_{n-1}^{n-1} y^{n-1}
\end{array}\right)
$$

where multiplication in the determinant is convolution. Denoting by $M_{i j}$ the 
minors of the determinant, we obtain that on $\square_{a_{n-1}}$

$$
\phi_{n}\left(g_{1}, \ldots, g_{n}\right)=\sum_{k=1}^{n}(-1)^{k} x^{k-1} \star M_{1 k} .
$$

The highest power of $x$ appears when $k=n$, namely, in the term $x^{n-1} \star M_{1 n}$. Therefore, to show that $\phi_{n}\left(g_{1}, \ldots, g_{n}\right) \not \equiv 0$, it suffices to show that $x^{n-1} \star$ $M_{1 n} \not \equiv 0$; but since $M_{1 n}$ is a polynomial, this is obviously true if $M_{1 n} \not \equiv 0$. So it suffices to prove that

$$
M_{1 n}=\operatorname{det}\left(\begin{array}{cccc}
1 & y & \cdots & y^{n-2} \\
1 & a_{2} y & \cdots & a_{2}^{n-2} y^{n-2} \\
\vdots & \vdots & & \vdots \\
1 & a_{n-1} y & \cdots & a_{n-1}^{n-2} y^{n-2}
\end{array}\right) \not \equiv 0
$$

It is easy to see that

$$
M_{1 n}=\operatorname{det}\left(\begin{array}{cccc}
1 & 1 & \cdots & 1 \\
1 & a_{2} & \cdots & a_{2}^{n-2} \\
\vdots & \vdots & & \vdots \\
1 & a_{n-1} & \cdots & a_{n-1}^{n-2}
\end{array}\right) U \star y \star \cdots \star y^{n-2}
$$

where the multiplication in the last determinant is the usual multiplication. But the last determinant is the Van-der-Monde determinant of $a_{1}=1, a_{2}, \ldots, a_{n-1}$ and hence, is equal to $\prod_{i>j \geq 1}\left(a_{i}-a_{j}\right)$, which is not zero since $a_{i}>a_{j}$, for $i>j$, so $M_{1 n} \not \equiv 0$.

This completes the proof of Proposition 3 and, hence, also of Theorem 1.

\section{THE REDUCING SUBSPACES OF $W$}

We recall that a subspace $E$ of $L_{2}[0,1]^{2}$ is reducing for an operator $T$ on $L_{2}[0,1]^{2}$ if $E$ and $E^{\perp}$ are both invariant under $T$, or equivalently if $E$ is invariant under $T$ and $T^{*}$. We denote by $S_{+}$the symmetric functions in $L_{2}[0,1]^{2}$, namely,

$$
S_{+}=\left\{f \in L_{2}[0,1]^{2}: f(x, y)=f(y, x) \text { a.e. on }[0,1]^{2}\right\},
$$

and by $S_{-}$the antisymmetric functions in $L_{2}[0,1]^{2}$, namely,

$$
S_{-}=\left\{f \in L_{2}[0,1]^{2}: f(x, y)=-f(y, x) \text { a.e. on }[0,1]^{2}\right\} \text {. }
$$

Consider the operator $\tau$ defined on $L_{2}[0,1]^{2}$ by

$$
(\tau f)(x, y)=f(y, x) \text {. }
$$

It is easily verified that $\tau$ commutes with $W$. Therefore, if $f \in S_{+}$then

$$
\tau(W f)=W(\tau f)=W f
$$

hence, $W f \in S_{+}$. Similarly if $f \in S_{-}$then $W f \in S_{-}$; that is, $S_{+}$and $S_{-}$ are invariant under $W$. It is easy to see that $S_{-}$is the orthogonal complement of $S_{+}$, and, therefore, $S_{+}, S_{-}$are reducing subspaces of $W$.

The main result of this section is the following theorem.

Theorem 4. The only nontrivial reducing subspaces of $W$ are $S_{+}$and $S_{-}$.

For the proof of the theorem we shall need several lemmas. 
Lemma 5. Let $T$ be an operator in a Hilbert space $\mathscr{H}$, and assume $M$ is a reducing subspace for $T$. If $\lambda$ is an eigenvalue of multiplicity one and $x a$ corresponding eigenvector, then $x \in M$ or $x \in M^{\perp}$.

Proof. Let $\lambda$ be an eigenvalue of multiplicity one and $x$ a corresponding eigenvector. Suppose $x=x_{1}+x_{2}$, where $x_{1} \in M$ and $x_{2} \in M^{\perp}$. Then

$$
\lambda x=T x=T x_{1}+T x_{2} .
$$

Since $M$ and $M^{\perp}$ are invariant under $T, T x_{1} \in M$ and $T x_{2} \in M^{\perp}$, hence by (7), $T x_{1}=\lambda x_{1}$ and $T x_{2}=\lambda x_{2}$. Since $\lambda$ is of multiplicity one, this implies that $x_{1} \equiv 0$ or $x_{2} \equiv 0$, hence $x \in M$ or $x \in M^{\perp}$.

A simple computation shows that the adjoint of $W$ is given by

$$
\left(W^{*} g\right)(t, s)=\int_{s}^{1} d y \int_{t}^{1} g(x, y) d x, \quad g \in L_{2}[0,1]^{2} .
$$

Lemma 6. For every integer $n \neq 0, r_{n}=i / 2 \pi n$ is an eigenvalue of multiplicity one of the operator $W-W^{*}$, and the corresponding eigenfunctions are constant multiples of the function $f_{n}(x, y)=e^{-2 \pi i n x}-e^{-2 \pi i n y}$.

Proof. Let $\lambda \neq 0$ be an eigenvalue of $W-W^{*}$ and $F(x, y)$ a corresponding eigenfunction. Then $\left(W-W^{*}\right) F=\lambda F$. This implies that

$$
\int_{0}^{y} d s \int_{0}^{x} F(t, s) d t-\int_{y}^{1} d s \int_{x}^{1} F(t, s) d t=\lambda F(x, y) .
$$

The left-hand side of $(8)$ is a continuous function, so $F$ is continuous, and therefore, the left-hand side is a differentiable function. Differentiating (8) with respect to $y$, we get that

$$
\int_{0}^{1} F(t, y) d t=\lambda \frac{\partial F}{\partial y} .
$$

Differentiating (9) with respect to $x$ we obtain the differential equation

$$
\lambda \frac{\partial^{2} F}{\partial x \partial y}=0 .
$$

From the assumption that $\lambda \neq 0,(10)$ implies that

$$
F(x, y)=f(x)+g(y),
$$

where $f$ and $g$ are differentiable functions on $[0,1]$. Substituting this in (9) we obtain that

hence,

$$
\int_{0}^{1}[f(t)+g(y)] d t=\lambda \frac{d g}{d y}
$$

$$
C_{1}+g(y)=\lambda \frac{d g}{d y},
$$

where $C_{1}=\int_{0}^{1} f(t) d t$. The solution of this differential equation is

$$
g(y)=B e^{y / \lambda}+C_{1},
$$

where $B$ is a constant. Similarly we obtain that $f(x)=A e^{x / \lambda}+C_{2}$, where $A$ and $C_{2}$ are constants, and therefore we obtain that for some constant $C$

$$
F(x, y)=A e^{x / \lambda}+B e^{y / \lambda}+C,
$$


and substituting again (13) in the equation $\left(W-W^{*}\right) F=\lambda F$, we obtain

$$
\begin{aligned}
& \int_{0}^{y} d t \int_{0}^{x}\left(A e^{t / \lambda}+B e^{s / \lambda}+C\right) d t-\int_{y}^{1} d s \int_{x}^{1}\left(A e^{t / \lambda}+B e^{s / \lambda}+C\right) d t \\
& =\lambda\left(A e^{x / \lambda}+B e^{y / \lambda}+C\right) .
\end{aligned}
$$

This implies that

$$
\begin{gathered}
y\left[-\lambda A+\lambda A e^{1 / \lambda}+C\right]+x\left[-\lambda B+\lambda B e^{1 / \lambda}+C\right] \\
+\left[-\lambda A e^{1 / \lambda}-\lambda B e^{1 / \lambda}-C-\lambda C\right] \equiv 0 ;
\end{gathered}
$$

hence, we obtain the following three equations:

$$
\begin{aligned}
& \text { (1) }-\lambda A+\lambda A e^{1 / \lambda}+C=0 . \\
& \text { (2) }-\lambda B+\lambda B e^{1 / \lambda}+C=0 . \\
& \text { (3) }-\lambda A e^{1 / \lambda}-\lambda B e^{1 / \lambda}-C-\lambda C=0 .
\end{aligned}
$$

By subtracting (2) from (1) we get $(B-A)\left(1-e^{1 / \lambda}\right)=0$.

Possibility a. $e^{1 / \lambda}-1=0$. The solutions are: $\lambda_{n}=i / 2 \pi n$ for a nonzero integer $n$ and then $C=0$ and $A=-B$; namely, the eigenvalues are $r_{n}=i / 2 \pi n$ and the corresponding eigenfunctions are constant multiples of the functions $f_{n}(x, y)=e^{-2 \pi i n x}-e^{-2 \pi i n y}$.

Possibility b. $A=B$. In this case, $C=-2 \lambda A /(\lambda-1)$ where $\lambda$ is the solution of the equation $e^{1 / \lambda}=(\lambda+1) /(\lambda-1)$. It is easily verified that $r_{n}=i / 2 \pi n$ is not a solution of the last equation. (The solutions of this equation give other eigenvalues, in which we are not interested here.) So for any $n \neq 0, r_{n}$ is an eigenvalue of multiplicity one and $f_{n}$ is a corresponding eigenfunction.

Lemma 7. Let $P_{k m}(x, y)=x^{k} y^{m}-x^{m} y^{k}$ and $Q_{k m}(x, y)=x^{k} y^{m}+x^{m} y^{k}$. Then:

(1) $\overline{\operatorname{span}}\left\{Q_{k m}(x, y), k \geq m\right\}=S_{+}$, and

(2) $\overline{\operatorname{span}}\left\{P_{k m}(x, y), k>m\right\}=S_{-}$.

Proof. Let $Q$ denote the set of symmetric polynomials in two variables-that is, $Q=\left\{q ; q(x, y)=q(y, x), \forall(x, y) \in[0,1]^{2}\right\}$-and $P$ the set of antisymmetric polynomials in two variables-that is, $P=\{p ; p(x, y)=-p(y, x)$, $\left.\forall(x, y) \in[0,1]^{2}\right\}$. It is easily seen that $Q$ is the linear span of the polynomials $Q_{k m}$ and $P$ is the linear span of the polynomials $P_{k m}$. This implies the lemma by observing that $Q$ is dense in $S_{+}$and $P$ is dense in $S_{-}$.

Lemma 8. If $M$ is a reducing subspace for $W$ then $S_{-} \subseteq M$ or $S_{-} \subseteq M^{\perp}$.

Proof. Since $M$ is a reducing subspace for $W$, it is also reducing for $W-W^{*}$. By Lemmas 5 and 6 we get that, for any $n \neq 0, f_{n}(x, y)=e^{-2 \pi i n x}-e^{-2 \pi i n y}$ belongs either to $M$ or to $M^{\perp}$. In particular, $f(x, y)=e^{2 \pi i x}-e^{2 \pi i y}$ belongs either to $M$ or to $M^{\perp}$.

We now show that if $f \in M$ then $S_{-} \subseteq M$. For every $n \geq 2$ consider the polynomial $P_{n}$ defined by

$$
P_{n}(x, y)=y^{n}-x^{n}+x-y .
$$

First we claim that $P_{n} \in M, n=1,2, \ldots$ We prove this by induction. Since

$$
f(x, y)=e^{2 \pi i x}-e^{2 \pi i y} \in M
$$


and $M$ is invariant under $W$ and $W^{*}$,

$$
f_{1}=4 \pi i W^{*} W f+\frac{1}{2 \pi i} f \in M
$$

and

$$
f_{2}=4 \pi i W W^{*} f+2 W f \in M .
$$

A direct computation shows that

$f_{1}(x, y)=x^{2}\left[\frac{1}{2 \pi i}-1-\frac{1}{2 \pi i} e^{2 \pi i y}+y\right]-y^{2}\left[\frac{1}{2 \pi i}-1-\frac{1}{2 \pi i} e^{2 \pi i x}+x\right]+(x-y)$

and

$$
f_{2}(x, y)=x^{2}\left[\frac{1}{2 \pi i}-\frac{1}{2 \pi i} e^{2 \pi i y}+y\right]-y^{2}\left[\frac{1}{2 \pi i}-\frac{1}{2 \pi i} e^{2 \pi i x}+x\right] .
$$

Therefore,

$$
f_{1}(x, y)-f_{2}(x, y)=x-y-x^{2}+y^{2}=P_{2}(x, y) \in M .
$$

A simple computation shows that

$$
P_{n+1}=(n+1)\left[W P_{n}-W^{*} P_{n}+\frac{1}{2} P_{2}\right],
$$

and therefore if we assume that $P_{n} \in M$, we obtain that also $P_{n+1} \in M$, and the claim is proved.

Next we claim that, for every $n \geq 1, x^{n}-y^{n} \in M$. Indeed, since $M$ is closed and

$$
\left\|P_{n}-(x-y)\right\|_{2}=\left\|y^{n}-x^{n}\right\|_{2} \leq\left\|y^{n}\right\|_{2}+\left\|x^{n}\right\|_{2}=2 \frac{1}{\sqrt{2 n+1}} \rightarrow \rightarrow \infty 0,
$$

we conclude that $x-y \in M$. Since for every $n \geq 2$

$$
P_{n}(x, y)=y^{n}-x^{n}+x-y \in M,
$$

this implies that for every $n \geq 1$

$$
x^{n}-y^{n} \in M
$$

and, therefore, for $k>m$

$$
W^{m}\left(x^{k-m}-y^{k-m}\right)=\frac{1}{m !(k-m+1)(k-m+2) \cdots k} P_{k m}(x, y) \in M .
$$

Hence $P_{k m} \in M$ and by Lemma 6 , this implies that $S_{-} \subseteq M$. Similarly one shows that if $f \in M^{\perp}$ then $S_{-} \subseteq M^{\perp}$.

For every pair of nonnegative integers $n, m \geq 0$ denote

$$
f_{n m}(x, y)=\cos \left(\frac{2 n+1}{2} \pi x\right) \cos \left(\frac{2 m+1}{2} \pi y\right) \text {. }
$$

Lemma 9. The only eigenfunctions of the operator $W^{*} W$ are constant multiples of the functions $\left\{f_{n m}\right\}_{n, m \geq 0}$, and the corresponding eigenvalues are $\lambda_{n m}=$ $16 /(2 n+1)^{2}(2 m+1)^{2} \pi^{4}$.

Proof. It is easy to verify that $f_{n m}$ are eigenfunctions of $W^{*} W$ and that $\lambda_{n m}$ are the corresponding eigenvalues. Since it is well known that $\left\{f_{n m}\right\}_{n, m \geq 0}$ is 
a complete orthogonal system in $L_{2}[0,1]^{2}$, it follows that there are no other eigenfunctions.

Lemma 10. If $M$ is a reducing subspace for $W$ then either $S_{+} \subseteq M$ or $S_{+} \subseteq$ $M^{\perp}$.

Proof. If $M$ is reducing for $W$ then $M$ is also reducing for $W^{*} W$. Lemma 9 implies that $\lambda=16 / \pi^{4}$ is an eigenvalue of multiplicity one of the operator $W^{*} W$, and a corresponding eigenfunction is $f(x, y)=\cos (\pi x / 2) \cos (\pi y / 2)$. Hence by Lemma $5, f$ belongs either to $M$ or to $M^{\perp}$.

We will show that if $f \in M$ then $S_{+} \subseteq M$. For every $n \geq 1$ define $g_{n}(x, y)=1-x^{n}-y^{n}$. First we claim that $g_{n} \in M$ for $n=1,2, \ldots$. We prove this by induction. Since $f(x, y)=\cos (\pi x / 2) \cos (\pi y / 2) \in M$ and $M$ is invariant under $W$ and $W^{*}$,

$$
f_{1}=\frac{\pi^{4}}{16} W^{2} f-f \in M
$$

and

$$
f_{2}=\frac{\pi^{3}}{4(\pi-2)}\left[\left(W^{*}\right)^{2} f-W W^{*} f\right] \in M .
$$

By a direct computation

$$
f_{1}(x, y)=1-\cos \left(\frac{\pi}{2} x\right)-\cos \left(\frac{\pi}{2} y\right)
$$

and

$$
f_{2}(x, y)=1+\frac{2}{\pi}\left[1-\cos \left(\frac{\pi}{2} x\right)-\cos \left(\frac{\pi}{2} y\right)\right]-(y+x) .
$$

This implies that

$$
g_{1}=f_{2}-\frac{2}{\pi} f_{1} \in M .
$$

A simple computation shows that

$$
g_{n+1}=(n+1)\left[\frac{n}{n+1} g_{1}-W^{*} g_{n}+W g_{n}\right] \text {. }
$$

Therefore, if we assume that $g_{n} \in M$, we obtain that also $g_{n+1} \in M$, and the claim is proved. It is easy to see that $g_{n} \rightarrow 1$ in $L_{2}[0,1]^{2}$, and therefore since $M$ is closed, the function $U \equiv 1$ belong to $M$. Since $g_{n} \in M$, this implies that, for every $n \geq 0, x^{n}+y^{n} \in M$, and therefore, for every $0 \leq m \leq k$,

$$
W^{m}\left(x^{k-m}+y^{k-m}\right)=\frac{1}{m !(k-m+1) \cdots k} Q_{k m} \in M .
$$

Hence $Q_{k m} \in M$, and by Lemma 7 we conclude that $S_{+} \subseteq M$. A similar argument shows that if $f \in M^{\perp}$ then $S_{-} \subseteq M^{\perp}$.

Proof of Theorem 4. Let $M$ be a reducing subspace for $W$. It follows from Lemmas 8 and 10 that there are four possibilities:

(1) $S_{-} \subseteq M$ and $S_{+} \subseteq M$.

(2) $S_{-} \subseteq M^{\perp}$ and $S_{+} \subseteq M^{\perp}$.

(3) $S_{-} \subseteq M$ and $S_{+} \subseteq M^{\perp}$.

(4) $S_{-} \subseteq M^{\perp}$ and $S_{+} \subseteq M$. 
Since $S_{-} \oplus S_{+}=L_{2}[0,1]^{2}$, possibility (1) implies that $M=L_{2}[0,1]^{2}$ and possibility (2) implies that $M^{\perp}=L_{2}[0,1]^{2}$; hence, $M=\{0\}$. Since $S_{+}^{\perp}=S_{-}$, possibility (3) implies that $M=S_{-}$and possibility (4) implies that $M=S_{+}$. This concludes the proof of the theorem.

\section{INVARIANT AND HYPERINVARIANT SUBSPACES FOR $W$}

It is easy to see that if $E$ is a measurable subset of $[0,1]^{2}$, which satisfies the condition

$$
(x, y) \in E \Rightarrow[0, x] \times[0, y] \subseteq E,
$$

then the subspace

$$
M_{E} \stackrel{\text { def }}{=}\left\{f \in L_{2}[0,1]^{2}: f=0 \text { a.e. on } E\right\}
$$

is an invariant subspace for $W$. These subspaces are in a sense analogous to the invariant subspaces of the classical Volterra operator $V$; however, there are many other invariant subspaces for $W$. For example, such are the subspaces $S_{+}$and $S_{-}$considered in $\S 3$; and if $G$ is any finite subset of $L_{2}[0,1]^{2}$, then in view of Theorem 1 the cyclic subspaces generated by $G$-that is, the closed span of the set $\left\{W^{n} f: f \in G, n \geq 0\right\}$-is a proper invariant subspace of $W$. In particular, if $G$ consists of the single function $U \equiv 1$, then it is easily verified that this subspace consists of all functions $f$ in $L_{2}[0,1]^{2}$, which are of the form $f(x, y)=g(x y)$, where $g$ is a measurable function on $[0,1]$.

These examples indicate that $W$ has a very rich and varied supply of invariant subspaces, and a characterization of all of them might be a hopeless task. On the other hand, it might be easier to characterize all the hyperinvariant subspaces of $W$.

First we note, that unlike for $V$, not every invariant subspace of $W$ is also a hyperinvariant subspace. Indeed, since the operator $\tau$ (introduced in $\S 3$ ) commutes with $W$, every hyperinvariant subspace for $W$ must be invariant for $\tau$. This implies, in particular, that a necessary condition for an invariant subspace of the form $M_{E}$ to be hyperinvariant is that $E$ should be a symmetric set (that is, if $(x, y) \in E$ then $(y, x) \in E$ for almost all $(x, y) \in E)$. Thus, for example, if $0<a, b<1$ and $a \neq b$ then the subspace $M_{[0, a] \times[0, b]}$ is an invariant subspace for $W$ which is not hyperinvariant.

It should be observed that not every invariant subspace of $W$ which is also invariant for $\tau$ is hyperinvariant for $W$. Such examples are provided by the subspaces $S_{+}$and $S_{-}$which are not hyperinvariant for $W$, since they are not invariant for the convolution operator defined on $L_{2}[0,1]^{2}$ by $L_{h} f=h \star f$, with $h(x, y)=x$, which commutes with $W$.

We conclude with two problems.

Problem 1. Let $E$ be a measurable subset of $[0,1]^{2}$ which satisfies

(1) $(x, y) \in E \Rightarrow[0, x] \times[0, y] \subseteq E$, and

(2) $(x, y) \in E \Rightarrow(y, x) \in E$.

Is $M_{E}$ a hyperinvariant subspace for $W$ ? In particular, is the answer positive if $E=[0, a]^{2}$ for some $0<a<1$ ?

Problem 2. Is every hyperinvariant subspace for $W$ of the form $M_{E}$, where $E$ is a subset as in Problem 1? 

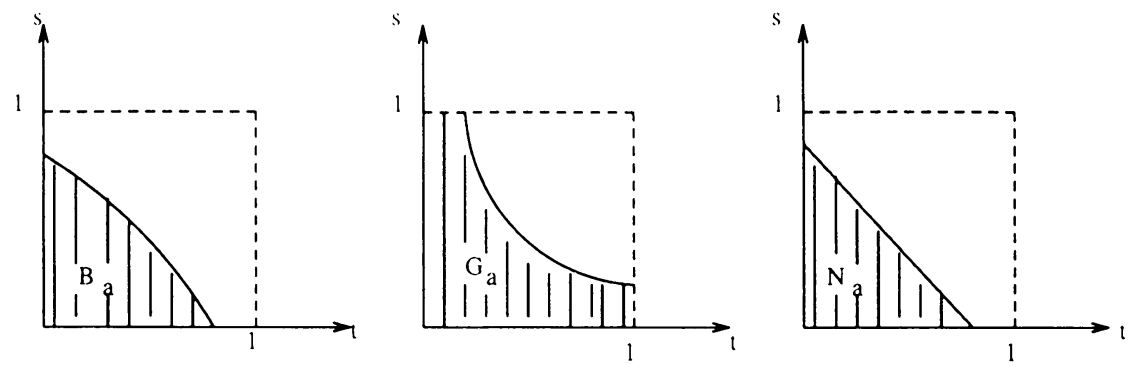

FIGURE 1. $M_{B_{a}}, M_{G_{a}}, M_{N_{a}}$ consist of all functions that vanish in the domains $B_{a}, G_{a}$, and $N_{a}$ respectively

We mention without proof that one can show that if for $0<a<1$ we denote $B_{a}=\left\{(t, s) \in[0,1]^{2}:(1-t)(1-s) \geq a\right\}, G_{a}=\left\{(t, s) \in[0,1]^{2}: t s \leq a\right\}$, and, for $0<a<2, N_{a}=\left\{(t, s) \in[0,1]^{2}: s+t \leq a\right\}$, then all the subspaces $M_{B_{a}}, M_{G_{a}}$, and $M_{N_{a}}$ are hyperinvariant subspaces for $W$. (See Figure 1.) Thus we obtain a positive answer to the first part of Problem 1 in these particular cases.

\section{REFERENCES}

1. A. Atzmon, Multilinear mappings and estimates of multiplicity, Integral Equations Operator Theory 10 (1987), 1-16.

2. H. Radjavi and P. Rosenthal, Invariant subspaces, Springer-Verlag, New York, 1973.

School of Mathematical Sciences, Raymond and Beverly Sackler Faculty of Exact Sciences, Tel-Aviv University, Tel-Aviv 69978, IsRael

E-mail address, A. Atzmon: aatzmon@math.tau.ac.il 